Ingenium. Revista Electrónica de Pensamiento Moderno y Metodología en Historia de la Ideas ISSN: 1989-3663

http://dx.doi.org/10.5209/rev_INGE.2016.v10.54741

\title{
La "conversación" sobre Blaise Pascal de Giuseppe Tomasi di Lampedusa
}

Alberto Mira Almodóvar ${ }^{1}$

Recibido: 25 de julio de 2016 / Aceptado: 16 de agosto de 2016

Resumen. Giuseppe Tomasi di Lampedusa nos ofrece en este escrito una exposición sobre la incidencia de la fuerza espiritual francesa del siglo XVII, con un esbozo de la historia y hechos acontecidos en Port-Royal des Champs y en el ambiente creado por el monasterio como centro del pensamiento francés de este siglo. Se completa la lección con un recorrido biográfico y por el pensamiento de Blaise Pascal, en el cual, como indica el propio Lampedusa, halló Port-Royal su mejor defensor y su máxima expresión.

Palabras clave: jansenismo, jesuitismo, duda, fe, miseria, grandeza, soledad, silencio.

\section{[en] Giuseppe Tomasi di Lampedusa’s “conversation” about Blaise Pascal}

Abstract. Giuseppe Tomasi di Lampedusa offers in this writing an exposition on the incidence of the French spiritual strength of seventeenth century, with an outline of the history and events in PortRoyal des Champs and the atmosphere created by the monastery as a center of French thought of this century. The lesson is complete with a tour by the biography and thought of Blaise Pascal, in which, as indicated by Lampedusa himself, Port-Royal found its best advocate and its maximum expression.

Keywords: Jansenism, Jesuitism, doubt, faith, misery, greatness, loneliness, silence.

Cómo citar: Mira Almodóvar, A. (2016) La “conversación” sobre Blaise Pascal de Giuseppe Tomasi di Lampedusa, en Ingenium. Revista Electrónica de Pensamiento Moderno y Metodología en Historia de la Ideas 10, 229-252.

\section{Europa en Sicilia: el placer de la palabra}

Giuseppe Tomasi di Lampedusa, príncipe de Lampedusa, descendiente de virreyes nombrados por los españoles, nacido en Palermo el 23 de diciembre de 1896, fallecido en Roma el 23 de julio de 1957, durante los últimos cuatro años de su vida dio voz a los escritos que había elaborado - y que siguió componiendosobre literatura occidental, principalmente inglesa y francesa de los siglos XVI al XIX, con un increíble y abierto eclecticismo, lo cual permite afirmar, con palabras

$1 \quad$ E-mail: amiraalmodovar@telefonica.net 
de su amigo Francesco Orlando ${ }^{2}$, que no existe escritor de alguna importancia en la historia de la literatura de estos siglos por cuyas cualidades Lampedusa no mostrara el interés del ingenuo disfrute del sabio.

En el verano de 1953 Lampedusa conoce al joven Francesco Orlando, estudiante de jurisprudencia, a través de su amigo el barón Pietro Sgadari di Lo Monaco (conocido entre sus amistades como Bebbuzzo), crítico musical del Giornale di Sicilia. Entre ambos nació una relación de amistad que se prolongó hasta el fallecimiento de Lampedusa. En ese mismo año de 1953 Lampedusa propuso a Orlando enseñarle la lengua y literatura inglesas, para lo cual acordaron tres encuentros semanales en la primera planta de la casa que Giuseppe Tomasi tenía en Via Butera 28, en Palermo. Comenzaron la labor con lecciones sobre la lengua inglesa pero pronto pasaron a las lecturas sobre la literatura, sin el más mínimo asomo didáctico sino con el deseo del disfrute y la conversación sobre ellas. Al poco tiempo, el auditorio se amplió con un pequeño grupo de jóvenes que Lampedusa había conocido a la vez que a Orlando - siempre por el mismo conducto del barón Pietro Sgadari-, y que eran aquellos que, junto al propio Orlando y a Francesco Agnello, cada mañana se dejaban ver alrededor de la mesa que en el café Mazzara ocupaba el príncipe siciliano desde las once horas hasta el mediodía. Los jóvenes asistentes eran: Antonio Pascualino y su hermana Beatrice, Gioacchino Lanza - el cual acabaría convirtiéndose en su hijo adoptivo-y su novia, Mirella Radice, a los que a veces se unía algún otro como Tito La Francesca, Ernesto Savagnone, Gabriella Saladino y el propio Bebbuzzo.

Hasta el otoño de 1954 leyeron y comentaron los escritos acerca de la literatura inglesa redactados por el príncipe, $\mathrm{y}$, según consta anotado a pie de página en su diario, a partir del 22 de enero de 1955 comenzaron la actividad de lectura sobre literatura francesa, con Orlando como único lector. Iniciaron estas sesiones con los poetas del siglo XVI, en honor de la «Pléiade», el grupo de poetas admiradores de la cultura greco-latina encabezados por Pierre de Ronsard y Joachim Du Bellay, cuya Défense et illustration de la langue française (abril de 1549) es considerada como su "manifiesto". Este grupo, como indica el propio Lampedusa en las páginas dedicadas a ellos, fue el principal impulsor del desarrollo y estandarización de la lengua francesa en el renacimiento literario. El resto de componentes iniciales de la «Pléiade» lo componían: Antoine de Baiff, Rémy Belleau, Étienne Jodelle, Pontus de Tyard y Jean Dorat. Casi cuatro siglos después, en 1931, el joven editor independiente Jacques Schiffrin, natural de Azerbaiyán, inspirado en este grupo de poetas funda la Bibliothèque de la Pléiade, como una colección especial de "Éditions de la Pléiade/J. Schiffrin \& Cie" (creada en 1923), para reunir obras completas de autores clásicos. Ante las dificultades de tesorería de Schiffrin, el 31 de julio de 1933 Gaston Gallimard aconsejado por André Gide y Jean Schlumberger, fundadores y administradores de la Nouvelle Revue Française $(N R F)$, integra en Éditions Gallimard la Bibliothèque de la Pléiade, manteniendo a Jacques Schiffrin en la dirección de esta colección hasta el comienzo de la II Guerra Mundial, en septiembre de 1939.

2 Francesco Orlando, Recuerdo de Lampedusa, Juan Antonio Méndez Borra (trad.), Valencia, Pre-Textos, 2006. 
Las ediciones de La Pléiade, que tanto amaba Lampedusa y de la cual poseía una colección actualizada, contribuyeron de manera notable al desarrollo y mantenimiento de ese particular diletantismo aristocrático desde el que el autor siciliano podía acercarse, casi descuidadamente, y penetrar en los textos literarios con sus convicciones acerca de lo explícito y lo implícito en la literatura; convencimiento tomado de la teoría del biografismo de su admirado Sainte-Beuve (no sólo por su gran obra sobre Port-Royal des Champs), de acuerdo con la cual la íntima y casi secreta explicación de las obras hay que buscarla en la vida de los autores.

Giuseppe Tomasi se enfrentaba a cualquier obra literaria con una actitud propensa hacia lo implícito en la misma, pues tenía cierta aversión hacia la excesiva explicitud. Como indica Francesco Orlando en su Recuerdo de Lampedusa, el maestro tendía a la lectura del máximo de cosas posibles entre líneas, más allá de la palabra escrita; cosas que estaban en el texto o que él consideraba que lo estaban pero las cuales, en cualquier caso, habían quedado casi ocultas por una voluntaria o involuntaria reserva del autor. Mas, naturalmente, no todos los escritores habían dedicado sus obras para "unos pocos afortunados". De ahí que Lampedusa repartía a los escritores entre «grassi» y «magri», sin que tuviera especial predilección por los del segundo grupo frente a los del primero, pues como le dijo a su joven amigo en una de las primeras ocasiones en las que participaban en una lectura sobre la cual estaba decayendo el interés: "¡Hay que saber aburrirse, Orlando!”. Para el príncipe, los «gordos», los explícitos, expresan todos los aspectos y todos los matices de cuanto dicen, quitándole al lector la responsabilidad de deducir y desarrollar por sí mismo a partir de sus palabras, porque en ellas ya está todo deducido y desarrollado. Los «flacos», por el contrario, se leen incorporando con satisfacción esa estimulante responsabilidad. En ellos, lo no dicho, sin ser menos preciso, es más jugoso que lo dicho, ya que el arte sabio y alusivo del escritor encauza hacia ello al lector perspicaz. "Gordos» eran Dante, Montaigne, Shakespeare o Balzac; «flacos» (casi todos franceses), La Rochefoucauld, Pascal, Racine, Mallarmé o André Gide. Mas no hay que olvidar que para Lampedusa el preferido entre los preferidos era Shakespeare, el gran explícito entre los explícitos.

Las "conversaciones" completas sobre literatura inglesa y francesa del autor de El gatopardo se encuentran editadas únicamente en idioma italiano en su Opere ${ }^{3}$. Traducidas al español sólo se han publicado las referidas a autores franceses del siglo $\mathrm{XVI}^{4}$. Con este trabajo se pretende cubrir una mínima parte de esa carencia presentando en bilingüe, italiano-español, la "conversación" dedicada por Lampedusa a uno de los autores franceses por el cual sentía la mayor admiración y el máximo respeto, y que él mismo registra como uno de esos pocos hombres «que ha nacido para la inmortalidad»: Blaise Pascal.

3 Giuseppe Tomasi di LAmpedusa, Opere, a cura di Nicoletta Polo, introduzione e premesse di Gioacchino Lanza Tomasi, Milano, Mondadori, 1995 (V edizione accresciuta e aggiornata I Meridiani aprile 2004).

4 GIUSEPPE TOMASI DI LAMPEDUSA, Conversaciones literarias (invitación a las letras francesas del siglo XVI), José Ramón Monreal (trad.), Barcelona, Editorial Bruguera, 1983. 
Giuseppe Tomasi di Lampedusa

\section{BLAISE PASCAL (1632-1662) \\ E BREVI ACCENNI A PORT- ROYAL}

Pascal, per molte persone, è il più grande dei francesi.

Non ho nessuna voglia di cacciarmi nel gioco poco proficuo e immensamente ridicolo di decretare diplomi di grandezza o di negare la sufficienza a scrittori il minore dei quali vale spiritualmente cento volte noi, povere scimmie.

(Ricordate Max Jacob? «Moi, qui ne suis pourtant qu'un babouin»)

Primo, secondo, terzo o quarto che sia, Blaise Pascal è certamente uno dei primi cento uomini che siano mai nati all'immortalità, uno, soprattutto, degli uomini che potrebbero essere «le plus grand témoignage que nous puissons donner de notre dignité», come disse un suo pari, se vi fosse alcuno cui questa testimonianza potesse interessare.

Il genio di Pascal ha un carattere unico. Non che egli non abbia caratteri che lo avvicinino ad altri; però in Pascal vi è sempre un elemento in più che lo distingue. Per esempio egli possiede il temperamento inquisitivo, la tendenza scientifica al dubbio di Montaigne; in più però egli possiede il vantaggio di ver risolto il dubbio. Ha la curiosità scientifica, la larga simpatia umana di Goethe; in più ha di suo l'aver potuto sintetizzare scienza ed umanitarismo in un più alto insieme; Pascal ha traversato le angosce fisiche e il tedio della vita come Leopardi; ma lo supera in questo, che angosce e tedio sono state da lui utilizzate (e non dico
Giuseppe Tomasi di Lampedusa

BLAISE PASCAL (1632-1662)

Y BREVES ALUSIONES A PORTROYAL

Pascal, para muchas personas, es el más grande de los franceses.

No tengo deseo alguno de caer en el perjudicial e inmensamente ridículo juego de establecer títulos de grandeza o de negar la suficiencia a escritores de los cuales el menor de ellos vale espiritualmente cien veces más que nosotros, pobres simios.

(¿Recuerdas a Max Jacob? «Yo, que no soy más que un babuino»)

Primero, segundo, tercero o cuarto qué tanto da, Blaise Pascal es ciertamente uno entre los primeros cien hombres que ha nacido para la inmortalidad; sobre todo uno de los hombres que pueden representar «el mayor testimonio que pueda darse de nuestra dignidad», si hubiera alguien al que este testimonio pudiera interesar, como dijo un igual suyo.

El genio de Pascal tiene un carácter único. No es que no hubiera en él caracteres que lo acerquen a otros, pero siempre hay un elemento más que lo distingue. Por ejemplo, posee el temperamento inquisitivo, la tendencia científica a la duda de Montaigne, pero, además, él posee la ventaja de ver resuelta la duda. Tiene curiosidad científica; la gran simpatía humana de Goethe; el haber podido sintetizar ciencia y humanitarismo en un conjunto de superior nivel. Pascal ha atravesado la angustia física y el tedio de la vida como Leopardi, mas lo supera en que utiliza (y no digo utilización para fines científicos, que esto también lo hizo Leopardi) la angustia y el tedio en su 
utilizzate a scopi scientifici, ché ciò anche Leopardi ha fatto) proprio in funzione vitale.

Per dirla più in breve, Pascal ci presenta il fenomeno rarissimo (forse unico) di un temperamento analitico di scettico che viene a sboccare nelle conclusioni sintetiche di un credente.

Pascal non ha posseduto la sua verità come dono venuto dal di fuori: se la è creata lui pezzo per pezzo, scartando questo materiale, accettando l'altro dopo averlo soppesato e cagliato. Non sua è la credenza nell' «ipse dixit» di chicchessia; non per niente era cartesiano sino al midollo delle ossa, matematico altissimo e indagatore scrupoloso dei fatti fisici. La differenza con gli altri che hanno indagato il buio è in questo: che egli la luce poté scoprirla.

Occorre dire che se vi è esempio di pensiero che possa scuotere l'eterno «ignorabimus» dello scettico, questo è quello di Pascal.

Da questa sua doppia natura (perfettamente fusa) oltre che dal suo straordinario talento di espressione, deriva il fatto che non un rigo di Pascal è invecchiato; i suoi dubbi iniziali, la sua fede conclusiva sono egualmente giovani.

Dopo aver detto tante parole inutili bisogna adesso occuparsi un po' della sua opera. Ma prima di far questo sarà necessario sfiorare l'imponente ambiente di Port-Royal che condizionò Pascal e trovò in lui il proprio difensore e la propria espressione massima.

\section{Un milligrammo di fatti su Port-Royal}

Port-Royal era una abbazia cistercense di monache, situata nelle vicinanze di Versailles, in quel che a propia función vital.

Para decirlo de manera muy breve, Pascal presenta el fenómeno rarísimo (quizás único) de un temperamento analítico de escéptico que viene a desembocar en las conclusiones sintéticas de un creyente.

Pascal no ha poseído su verdad como un don venido de fuera, sino que la ha creado pieza por pieza, desechando este material, aceptando el otro después de haberlo sopesado $\mathrm{y}$ cuajado. La suya no es la creencia en «él lo dijo» de cualquier persona: no por nada era cartesiano hasta la médula de los huesos, altísimo matemático e investigador escrupuloso de los hechos físicos. La diferencia con otros que han indagado lo oscuro es ésta: que él era capaz de descubrir la luz.

Hay que decir que si existe un ejemplo de pensamiento que pueda agitar el eterno «lo ignoraremos» del escéptico, ese es el de Pascal.

De su naturaleza dual (perfectamente fundida) y de su extraordinario talento de expresión se deriva el hecho de que ni una línea de sus escritos haya envejecido; sus dudas iniciales, así como su concluyente fe se muestran igualmente jóvenes.

Después de haber dicho tantas palabras inútiles, ahora es preciso ocuparse un poco de su obra. Sin embargo, antes de hacer esto será necesario desentrañar el imponente ambiente de Port-Royal, el cual condicionó a Pascal y halló en él a su mejor defensor y su expresión máxima.

Un miligramo de hechos sobre PortRoyal

Port-Royal era una abadía cisterciense de monjas, situada en los alrededores de Versalles, en lo que nos 
noi postromantici appare un gradevole valloncello ombroso, proprio al margine della strada nazionale ParigiDreux; nei seicento questo posto appariva straordinariamente selvaggio ed incolto e veniva catalogato sotto l'etichetta di «désert affreux».

Il valloncello è solitario; fra le alte erbe ed all'ombra dei bei faggi si scorgono pochi frammenti di edifici: l'abbazia venne scoperchiata e demolita dai dragoni di Luigi XIV, scatenati in quel luogo dall'inespiabile rancore della Compagnia di Gesù.

Il «vallon» di Port-Royal, deserto e rinselvatichito com'è, deve essere annoverato fra $i$ più alti santuari dello spirito umano, come il Partenone, come Firenze, come Parigi lì d'accanto, come la grigia casa di Weimar.

Attorno a questo monastero che fu sempre poverissimo come fu sempre altero s'impernia e gira tutto il pensiero francese del Seicento: non vi è grande figura di quel tempo che ad esso non si annodi, come discepolo o come avversario, talvolta come devoto e nemico nello stesso tempo, come successe a Racine.

Corneille, Rotrou, La Rochefoucauld, Molière, Bossuet, Fénelon, Mme de Sévigné, La Bruyère, Saint-Simon, infiniti minori, e poi San Francesco di Sales, Luigi XIV, Colbert, Louvois, la Maintenon scorsero in questo sparuto convento un faro o uno scoglio. Per non parlare di Pascal o di Racine che sono i veri «genii loci».

Ma la forza spirituale di PortRoyal è tanto grande da estendere la sua attrazione al passato e all'avvenire: Montaigne si illumina di nuova luce se considerato in rapporto parece un agradable valle umbroso post-romántico, justo al lado de la carretera nacional París-Dreux. En el siglo diecisiete este lugar era extraordinariamente salvaje y baldío y estaba catalogado con la etiqueta de «horrendo desierto».

El valle es solitario; entre la alta hierba y la sombra de las hermosas hayas se ven pocos restos de edificios: la abadía fue vaciada y demolida por el cuerpo de dragones de Luis XIV, brotando en aquel lugar el inexpiable rencor de la Compañía de Jesús.

El «pequeño valle» de PortRoyal, desértico y selvático como es, debe contarse entre los más nobles santuarios del espíritu humano, como el Partenón, Florencia, París, allí al lado, o la casa gris de Weimar.

En torno a este monasterio, que fue siempre tan pobre como altivo, se articula y gira todo el pensamiento francés del siglo XVII: no hubo ni un gran personaje de aquel tiempo que no se relacionara con él, como discípulo o como adversario, en ocasiones como devoto y enemigo al mismo tiempo, como ocurrió con Racine.

Corneille, Rotrou, La Rochefoucauld, Molière, Bossuet, Fénelon, Mme de Sévigné, La Bruyère, Saint-Simon, más otros incontables menores; y luego también San Francisco de Sales, Luis XIV, Colbert, Louvois, la Maintenon vieron en este reducido convento un faro o un escollo, por no mencionar a Pascal o a Racine que son el verdadero «espíritu tutelar del lugar».

Mas la fuerza espiritual de PortRoyal es tanto más grande por extender su atracción al pasado y al futuro: Montaigne se ilumina nuevamente cuando es considerado en relación con 
alle «bonnes filles», Rousseau, SainteBeuve e Mauriac ne sono stati feriti.

Questo basterà a farvi capire come tentare qui una sia pur sommaria storia di Port-Royal e del movimento spirituale che in lui si incarnò, sia totalmente al di fuori delle possibilità obiettive di tempo. Quindi vi esporrò i fatti puri e semplici; se vorrete saperne di più potete leggere Sainte-Beuve che nel suo Port-Royal ha scritto il proprio capolavoro e forse quello della critica di tutti $\mathrm{i}$ tempi, ed i circa ventimila altri volumi che su questo argomento sono stati composti in tutti $\mathrm{i}$ paesi, senza esaurirlo.

Questa abbazia era stata fondata nel 1200. E per quattro secoli aveva vivacchiato santamente senza un particolare lustro. Alla fine delle guerre di religione essa si trovò devastata non soltanto materialmente ma anche dal punto di vista spirituale: molte suore si erano allontanate, quelle che restavano adempivano con fiacchezza e noia gli obblighi derivanti dai loro voti.

Nel 1608 venne eletta abbadessa la giovane mère Angélique, figlia del magistrato Arnauld, una delle più alte figure di donna di tutta la storia.

Nello stesso periodo di tempo, Jansénius, il vescovo di Ypres, nelle Fiandre, andava svolgendo un'attività religiosa meritoria ma singolare: nel quadro della Controriforma, dichiarandosi perfettamente ligio alle dottrine cattoliche, egli veniva scartando l'autorità di tutti i Padri della Chiesa e di tutte le decisioni dei Concili, e accettava come dottore infallibile Sant' Agostino. Egli formò accanto a sé un circolo di discepoli, las «buenas hijas» ${ }^{5}$; Rousseau, SainteBeuve y Mauriac han sido fuertemente conmovidos.

Esto bastará para hacer entender que está totalmente fuera de las posibilidades objetivas del tiempo el hecho de tratar aquí una historia de Port-Royal y del movimiento espiritual que en él se encarnó, aunque fuera breve. Por lo tanto, voy a explicar los hechos puros y simples. Si quieren saber más pueden leer a Sainte-Beuve, que en su Port-Royal ha escrito su obra maestra y quizás la de la crítica de todos los tiempos, además de los cerca de otros veinte mil volúmenes compuestos sobre este tema en todos los países, sin llegar a agotarlo.

La abadía se fundó en el año 1200. Durante cuatro siglos había sobrevivido santamente sin particular aureola. Al final de las guerras de religión se vio devastada, no sólo materialmente sino también desde un punto de vista espiritual: muchas hermanas se habían alejado, y aquellas que permanecieron cumplían con flojedad y aburrimiento las obligaciones derivadas de sus votos.

En 1608 es elegida abadesa la joven madre Angélique, hija del magistrado Arnauld, una de las más importantes figuras de mujer de toda la historia.

En este período de tiempo, Cornelio Jansenio, el obispo de Ypres, Flandes, estaba llevando a cabo una actividad religiosa meritoria pero singular: en el contexto de la Contrarreforma, y declarándose totalmente fiel a las doctrinas católicas, venía descartando la autoridad de todos los Padres de la Iglesia y de todas las decisiones de los Concilios, aceptando como doctor infalible de la Iglesia a san

5 En referencia a las "mesdames" de Port-Royal des Champs. 
tutti uomini di alto intelletto e di assoluta purezza di costumi che lentamente incominciarono a propagare i suoi insegnamenti. Uno di questi discepoli fu Du Vergier de Hauranne, passato alla storia sotto il nome di «abbé de Saint-Cyran».

Questi divenne il direttore spirituale del rinnovato Port-Royal e l'intero convento aderì alle dottrine di Giansenio.

Che cosa erano queste dottrine che il vescovo di Ypres aveva derivato da Sant'Agostino? In pochissime parole queste: non si può essere salvati se non mediante la Grazia. E questa Grazia è predeterminata fin dall'eternità, mediante criteri divini assolutamente imperscrutabili all'ingegno umano. Per così dire gli uomini, la maggior parte degli uomini, è dannata già prima di nascere. Le varie virtù e l'osservanza dei dettami della Chiesa sono sì obbligatorie perché ribellarvisi sarebbe ribellarsi alla volontà divina, ma non efficaci se non sono sorrette dalla Grazia che nessuno sa se la ha.

Questa dottrina, che è naturalmente infinitamente più complessa di quanto può apparire dalle precedenti dieci righe, si riallacciava alla dottrina di Lutero ed a quella di Calvino (benché vi fossero delle sensibili differenze che $i$ giansenisti tenevano a mettere bene in luce) e accontentava le anime più austere e più realmente religiose fra $i$ cattolici, nelle quali la Riforma aveva lasciato tracce indelebili e che erano ostili agli accomodamenti ed al sistema di «riduzioni di viaggio» per il Paradiso che erano sostenuti dai gesuiti.
Agustín. Formó junto a sí un círculo de discípulos: todos hombres de alto intelecto y absoluta pureza de costumbres, que lentamente comenzaron a propagar sus enseñanzas. Uno de estos discípulos fue Jean du Vergier de Hauranne ${ }^{6}$, que ha pasado a la historia con el nombre de «abad de Saint-Cyran», el cual se convirtió en el director espiritual del renovado PortRoyal y todo el monasterio se adhirió a las doctrinas de Jansenio.

¿Cuáles fueron estas doctrinas que el obispo de Ypres había derivado de san Agustín? En pocas palabras: que no es posible ser salvado si no es mediante la Gracia. Y que esta Gracia está predeterminada desde la eternidad, mediante criterios divinos absolutamente inescrutables al ingenio humano. Por así decir, los hombres, la mayor parte de los hombres, están condenados incluso antes del nacimiento. Las diversas virtudes y el cumplimiento de los dictados de la Iglesia sí son obligatorios, porque rebelarse contra ellos sería rebelarse contra la voluntad divina, mas no son eficaces si no están soportados por la Gracia que nadie sabe si se da.

Esta doctrina, que es desde luego infinitamente más compleja de cuanto puede aparecer en las diez líneas precedentes, vinculada a la doctrina de Lutero y de Calvino (aunque con sensibles diferencias que los jansenistas mantenían para mostrarlas bien a las claras), contentaba las almas más austeras y más religiosas entre los católicos -en las cuales la Reforma había dejado huellas indelebles- que eran hostiles a los arreglos y al sistema de «reducciones de viaje» hacia el Paraíso propugnado por los jesuitas.

También podemos encontrarlo escrito como Jean du Verger de Hauranne, tal como indica Sainte-Beuve en su Port-Royal-I, Paris, Bibliothèque de la Pléiade, 1952, p. 305: «Jean du Vergier ou du Verger de Hauranne, qui fut abbé de Saint-Cyran». 
Le conseguenze non soltanto religiose ma anche politichi e sociali di un diffondersi di questi insegnamenti erano incalcolabili: l'autorità della Chiesa cattolica sarebbe stata destinata a perire, se tutte le decisioni dei Concili, tutte le opere sacramentali e le osservanze fossero state dichiarate vuote di significato se non protocollare. La Riforma risorgeva non già dal di fuori, come era stato il caso di Lutero e di Calvino, ma dal di dentro, in modo più pericoloso perché più inafferrabile.

Nel 1640, dopo la morte la di Giansenio, venne pubblicata la sua opera principale, l'Augustinus, che consisteva di cinque colossali volumi latini «in folio». I gesuiti vi saltarono sopra, li spedirono a Roma e chiesero che la Santa Sede li condannasse. Il Vaticano non li condannò ma ne estrasse venticinque proposizione contenenti idee eretiche, condannò queste e chiese a tutti $\mathrm{i}$ vescovi e a tutti i religiosi di Francia di firmare una dichiarazione che accettasse la condanna.

A questo punto scoppiò la tempesta. L'enorme maggioranza firmò; alcuni vescovi non firmarono sostenendo che le venticinque proposizioni biasimate non si trovavano nell'opera di Giansenio, che esse erano state dedotte in mala fede da alcune pagine innocue ad opera dei gesuiti; che se vi fossero state ne avrebbero volentieri firmato la condanna, ma poiché non c'erano non potevano condannare il nulla.

Il monastero di Port-Royal non volle firmare senz'altro. Non ammetteva che si imponessero alla coscienza individuale dei vincoli. Ubbidientissime, reverentissime figliole della Chiesa: ma la credenza
Las consecuencias, no sólo religiosas sino también políticas $\mathrm{y}$ sociales de la difusión de estas enseñanzas, eran incalculables: la autoridad de la Iglesia Católica estaría destinada a perecer si todas las decisiones de los Concilios, todas las obras sacramentales y el cumplimiento de todas las obligaciones fueran declaradas vacías de significado y únicamente protocolarias. La Reforma resurgió no desde fuera, como había sido en el caso de Lutero y Calvino, sino desde dentro, de un modo más peligroso por ser menos controlable.

En 1640, después de la muerte de Jansenio, se publica su principal obra, Augustinus, compuesta por cinco grandes volúmenes en latín «in folio». Los jesuitas se adelantaron y enviaron la obra a Roma pidiendo a la Santa Sede su condena. El Vaticano no la condenó, pero extrajo veinticinco proposiciones con ideas consideradas heréticas que sí condenó, conminando a continuación a todos los obispos y religiosos de Francia a firmar una declaración de aceptación de esta sentencia.

En este punto estalló la tempestad. La enorme mayoría firmó. Algunos obispos no la firmaron sosteniendo que las veinticinco proposiciones consideradas heréticas no se encontraban en la obra de Jansenio: que habían sido deducidas de mala fe por los jesuitas de algunas páginas inocuas de la misma, y que si no hubiera sido así hubieran firmado con gusto la condena, mas como no había nada no podían condenar la nada.

Sin duda, el monasterio de PortRoyal no quería firmar. Pero no admitía que se impusieran a la conciencia individual los vínculos monacales. Las reverendísimas hijas de la Iglesia eran muy obedientes, mas la creencia individual no podía ser forzada. 
individuale non poteva essere sforzata.

Atto altamente rivoluzionario, compiuto da un pugno di umili religiose, guidate dal temperamento di fuoco della «mère Angélique».

Naturalmente, dopo numerosi e patetici interventi dell'arcivescovo di Parigi che costituiscono uno dei più elevati drammi spirituali, il monastero venne sciolto, le suore riprese in altri conventi.

Ma la vittoria dei gesuiti fu una vittoria di Pirro: il movimento giansenista aveva avuto ripercussione nulla sul clero secolare, scarsa sul clero regolare (una parte dei padri oratoriani fu sola ad appoggiare le intrepide ribelli di Port-Royal) ma vastissima sul laicato.

Numerosissime personalità appartenenti alle cassi più alte della società, tanto per influenza sociale come per cultura, tutti coloro, insomma, che ancora sentivano il cristianesimo come dramma e non come commedia a lieto fine, come predicavano $i$ gesuiti, avevano più $o$ meno palesemente aderito al giansenismo; molti avevano addirittura abbandonato le loro occupazioni mondane e, costruendosi delle casette nei pressi di Port-Royal, si erano ritirati, da laici, a far vita solitaria, devota e studiosa. Furono i cosiddetti «solitaires», «ces Messieurs de Port-Royal», che rinnovavano in piena epoca moderna $i$ fasti del cenobitismo dei primi secoli cristiani.

Così fitta era la popolazione di uomini dotti attorno al convento che venne spontanea l'idea di crearvi delle scuole per ragazzi, scuole che, dato il sapere degli insegnanti e la straordinaria modernità dei metodi
Acto altamente revolucionario, realizado por un puñado de humildes religiosas encabezadas por el temperamento de fuego de la «madre Angélique».

Naturalmente, después de numerosas y patéticas intervenciones del arzobispo de París, que constituyen uno de los más altos dramas espirituales, el monasterio fue disuelto y sus monjas repartidas en otros conventos.

Mas la victoria de los jesuitas fue una victoria pírrica: el movimiento jansenista había tenido una repercusión nula en el clero secular, escasa en el clero regular (sólo una parte de los padres del oratorio apoyaron a las intrépidas madres rebeldes de PortRoyal), pero amplísima entre los laicos.

Numerosas personalidades pertenecientes a las clases más altas de la sociedad, tanto por su influencia social como cultural, todos aquellos que, en definitiva, todavía sentían el cristianismo como drama y no como una comedia de final feliz, como predicaban los jesuitas, tenían más o menos clara su adhesión al jansenismo; muchos, incluso abandonando sus ocupaciones mundanas, se construyeron pequeñas casas en las cercanías de Port-Royal y allí, como laicos, se retiraron para llevar una vida solitaria, devota y de estudio. Fueron considerados «los solitarios», «los señores de Port-Royal», que renovaron en plena época moderna los fastos del cenobitismo de los primeros siglos del cristianismo.

La población de hombres doctos en torno al convento alcanzó tal densidad que surgió espontáneamente la idea de crear escuelas para los niños ${ }^{7}$; escuelas que, dado el conocimiento de los enseñantes y la extraordinaria modernidad de sus métodos pedagógicos,

7 Las conocidas como "Petites Écoles". 
pedagogici, divennero le migliori di Francia. In esse fu educato il giovinetto Racine.

Naturalmente le scuole vennero chiuse quando il monastero fu sciolto; ma molti dei «solitaires» seguirono le loro direttrici spirituali nelle più remote province francesi quando esse furono invitate al confino.

Questo esilio volontario di una cospicua parte della intellettualità francese commosse grandemente l'opinione pubblica ed i gesuiti divennero sempre meno popolari.

D'altronde la Compagnia stessa si trovava, malgrado la propria vittoria, in crisi. Erano state allora tradotte in francese le opere di casistica: dei manuali per i confessori nei quali $i$ più rari e sottili peccati vengono analizzati, scomposti in parti e scrutati per diagnosticarne la maggiore $\mathrm{o}$ minore perversità. Famoso fra questi casuisti era il padre gesuita Escobár, che era del resto una santa e degna persona.

Però, fedele alle direttive della Compagnia che voleva, come essa stessa diceva, «bâtir un chemin de velours pour arriver aux paradis» accumulava sofismi su sofismi per permettere ai confessori di assolvere peccati davvero ributtanti.

Lo scandalo fu immenso. Non si parlava che di questo. Fu allora che Pascal scrisse le sue Lettres provinciales nelle quali, con una dottrina illimitata ma ancora inferiore allo spirito caustico e alla indignazione morale, prendeva in giro e additava educatamente al disprezzo pubblico le teorie gesuitiche. Les provinciales, pubblicate senza nome di autore e stampate alla macchia, ebbero un successo incredibile. Dalla loro pubblicazione prende data la fama poco simpatica della Compagnia se convirtieron en las mejores de Francia. En ellas fue educado el joven Racine.

Naturalmente las escuelas se cerraron cuando el monasterio fue disuelto, mas muchos de «los solitarios» siguieron sus orientaciones espirituales en las más remotas provincias francesas a partir de su confinamiento.

Este exilio voluntario de una parte significativa de la intelectualidad francesa conmovió en alto grado a la opinión pública y los jesuitas se hicieron cada vez menos populares.

Por otra parte, la misma Compañía de Jesús, a pesar de su victoria, se hallaba en crisis. Fueron entonces traducidas al francés las obras de casuística: manuales para confesores en los cuales son analizados los pecados más raros y más sutiles, descompuestos en partes y escrutados para diagnosticar la mayor o menor perversidad de los mismos. Famoso entre estos casuistas era el padre jesuita Antonio Escobar, que, por lo demás, era una santa y digna persona.

No obstante, fiel a las directrices de la Compañía, la cual quería - como ella misma decía - «construir un camino de terciopelo para llegar al paraíso», acumulaba sofismas sobre sofismas para permitir al confesor la absolución de los pecados más repugnantes.

El escándalo fue inmenso. No se hablaba de otra cosa. Fue entonces cuando Pascal escribió sus Cartas provinciales en las cuales, con una doctrina sin restricciones pero aún inferior al ingenio cáustico y a la indignación moral, se burlaba y educadamente exponía al desprecio público las teorías jesuíticas. Las provinciales, publicadas sin nombre de autor e impresas en multicopista, tuvieron un éxito increíble. De su publicación data la fama antipática de 
di Gesù. Successo quanto mai meritato perché esse sono il capolavoro della letteratura polemica nel quale Pascal, già infermo e prossimo alla morte, si dimostrava schermidore abilissimo e creatore di una serie di piccole commedie spirituali del più alto valore artistico.

Intanto la situazione mutava, a favore di Port-Royal. Non soltanto l'indignazione creata dallo scioglimento del convento (contro il quale mai era stato possibile elevare il minimo sospetto di manchevolezze morali) aveva risvegliato l'opinione pubblica, ma anche necessità politiche avevano allontanato Luigi XIV da troppo intime relazioni con Roma; e ciò, oltre al prevalere del gallicanesimo che, benché acerrimo nemico del giansenismo in campo teologico, era d'accordo con questo nella immediata politica ecclesiastica, portò ad un ristabilimento del convento di Port-Royal da parte del re ed al ritorno degli esiliati al loro beneamato «désert».

$$
\text { L'odissea di Port-Royal era }
$$
però lungi dall'essere finita: polemiche accanite continuarono a svolgersi fra «le grand Arnauld»e Nicole da un lato, campioni dei giansenisti, e gli innumerevoli scrittori e scrittorucoli che i gesuiti scatenavano contro quel convento che era un rimprovero (non si più dire però «muto rimprovero») contro la progressiva «mondanizzazione» della vita spirituale. Finché i gesuiti ripresero forza (la carica di confessore del re era sempre assegnata a un gesuita) e finirono con l'ottenere la definitiva dispersione del convento e financo la sua materiale demoliziones che venne eseguita «manu militari».

Perfino il cimitero di PortRoyal venne distrutto e gli ossami di la Compañía de Jesús. Éxito tanto más meritorio pues supone la obra maestra de la literatura polémica, en la cual Pascal, ya enfermo y próximo a la muerte, demostró ser un esgrimista habilísimo y creador de una serie de pequeñas comedias espirituales del más alto valor artístico.

Mientras tanto la situación cambiaba a favor de Port-Royal. No sólo la indignación creada por la supresión del convento (contra el cual nunca fue posible suscitar la menor sospecha de faltas morales) había despertado la opinión pública, sino que también ciertas necesidades políticas imposibilitaban que Luis XIV tuviera relaciones demasiado íntimas con Roma; y todo esto, junto al predominio del galicanismo que, aunque acérrimo enemigo del jansenismo en el campo teológico, estaba de acuerdo con respecto a la inmediata política eclesiástica, llevó a un restablecimiento del convento de Port-Royal por parte del rey y al retorno de los exiliados a su muy amado «desierto».

La odisea de Port-Royal, sin embargo, estaba lejos de llegar a su fin: una fuerte polémica continuó desarrollándose entre «el gran Arnauld» y Nicole de un lado — grandes defensores del jansenismo-, y los innumerables escritores y aspirantes a serlo que los jesuitas incitaban contra aquel convento que era un reproche (no sé si decir, sin embargo, «mudo reproche») contra la progresiva «mundanización» de la vida espiritual. Mientras, los jesuitas retomaron fuerza (el cargo de confesor del rey siempre fue asignado a un jesuita) y terminaron por conseguir la definitiva dispersión del convento e incluso su material demolición que fue ejecutada «con el empleo de la fuerza armada».

Incluso el cementerio de PortRoyal fue destruido y los huesos de 
quegli alti esemplari di umanità versati alla fossa comune; fra questi gli avanzi di Racine che aveva chiesto di riposare insieme ai suoi maestri, spesso da lui vituperati ma sempre ricordati.

Durante il Settecento il giansenismo visse di una vita semiclandestina; andò via via affievolendosi e scomparve del tutto ai primi dell'Ottocento non senza che l'abbé Grégoire, ultimo quasi di quella indomabile setta, riuscisse, come deputato della Législative, a far cancellare l'antico ordinamento ecclesiastico francese.

Questa che avete udito è una specie di caricatura della storia di Port-Royal ed evidentemente non posso averne reso il senso di alto interesse spirituale che da esso fluisce.

Vi sarà sufficiente però per poter inquadrare la figura di Pascal nelle lotte del suo tempo.

Blaise Pascal era nato a Clermont-Ferrand, in Auvergne, il 19 giugno 1623. Il padre era un alto magistrato, assai dotto in scienze matematiche ed in fisica. Trasferitosi a Parigi nel 1631 egli fu il solo educatore del figlio, adoperando il metodo, strano in se stesso ma, per caso, adattissimo al formidabile ingegno del figlio, «de lui imposer des tâches toujours au-dessus de lui»». Così fin dalla prima infanzia la vita intellettuale di Pascal si atteggiò a quel tracciato di spirale sempre ascendente che costituì la sua sofferenza e la sua gloria.

Tutti conoscono i vari aneddoti sulla straordinaria precocità di Pascal; come a dodici anni egli riscoprisse da sé, senza l'aiuto di nessuna lettura, le prime trentadue proposizione geometriche di Euclide; come a sedici anni redigesse un trattato sulle sezioni aquellos altos ejemplares de la humanidad vertidos a la fosa común. Entre estos restos se encuentran los de Racine, que había pedido descansar al lado de sus maestros, a menudo por él vituperados mas siempre recordados.

Durante el siglo XVIII el jansenismo llevó una vida semiclandestina; se fue debilitando paulatinamente y desapareció por completo a principios del siglo XIX, no sin que el abate Gregorio -el último, casi, de aquella indómita secta - lograra, como diputado de la Convención Nacional, la cancelación del antiguo ordenamiento eclesiástico francés.

Esto que habéis oído es una especie de caricatura de la historia de Port-Royal, y evidentemente no he podido emitir el sentido del alto interés espiritual que de él fluye.

Será suficiente, sin embargo, para poder encuadrar la figura de Pascal en las luchas de su tiempo.

Blaise Pascal nació en ClermontFerrand, Auvergne, el 19 de junio de 1623. Su padre era un alto magistrado, muy docto en ciencias matemáticas y en física. Se trasladó a París en 1631, convirtiéndose en el único educador de su hijo. Adoptó el método, extraño en sí mismo pero adecuado al formidable ingenio del vástago, «de imponerle unas tareas siempre por encima de él». Así, desde la primera infancia, la vida intelectual de Pascal se atiene a ese trazado en espiral siempre ascendente que constituyó su sufrimiento y su gloria.

Todos conocen las diversas anécdotas sobre la extraordinaria precocidad de Pascal, como que a los doce años descubrió por sí mismo, sin ayuda de ninguna lectura, las primeras treinta y dos proposiciones de la geometría de Euclides; o que a los dieciséis elaboró un tratado sobre las secciones cónicas 
coniche che fece lo stupore di Descartes; e come inventasse a venti anni la prima macchina calcolatrice a poco dopo per proprio conto e senza averne avuto previo sentore $\mathrm{i}$ famosi esperimenti sul vuoto di Torricelli.

A venticinque anni Pascal era famoso come uno dei primi matematici del suo tempo, il che non è poco dire, dato il grande fervore matematico di quegli anni in Francia e in tutta Europa (ed anche in Sicilia con G.B. Odierna), anni che appunto videro, con la collaborazione di Pascal, la creazione del moderno calcolo infinitesimale.

È superfluo insistere sull'importanza di questa formazione rigorosamente scientifica di Pascal che valorizza e sottolinea tutta la sua successiva evoluzione spirituale.

Perché la vita di Pascal è una continua ascesa spirituale, nella quale ogni gradino è conquistato con aspra lotta. Al principio di questa ascensione lo vediamo compiere un'azione vilissima che non voglio neppur riferire; due giorni prima della morte uscì volontariamente da casa sua per cederla a un povero diavolo di «sfrattato». Conquista della santità che è ben più ammirevole delle santità possedute fin dalla nascita.

Si suol parlare a torto della prima, della seconda, della terza conversione di Pascal: non vi sono svolte brusche in questa vita ma un continuo purificarsi, accelerato soltanto, non condizionato, da alcuni avvenimenti esteriori.

In questo suo processo di elevazione egli fu grandemente aiutato dalla sorella minore, Jacqueline, che finì monaca a PortRoyal e che dalle sue azioni e dalla lettere rimasteci si dimostra pari in dolorosa comprensività al suo illustre que provocó el estupor de Descartes; así como que inventase a los veinte años la primera máquina calculadora $\mathrm{o}$ poco después por su cuenta y sin haber tenido previo indicio los famosos experimentos sobre el vacío de Torricelli.

A los veinticinco años Pascal era famoso como uno de los primeros matemáticos de su tiempo; que no es decir poca cosa, dado el gran fervor matemático existente en esos años tanto en Francia como en toda Europa (y también en Sicilia con Gianbattista Odierna), años que precisamente vieron, con la colaboración de Pascal, la creación del moderno cálculo infinitesimal.

Es superfluo insistir acerca de la importancia de esta formación rigurosamente científica de Pascal que valoriza y subraya toda su posterior evolución espiritual.

Porque la vida de Pascal es una continua ascensión espiritual, en la que cada nuevo paso es conquistado en enconada lucha. Al principio de esta ascensión lo vemos realizar una acción vil a la cual ni siquiera quiero referirme. Mas dos días antes de su muerte salió voluntariamente de su casa para cedérsela a un pobre diablo «sin hogar». Conquista de la santidad que es aún más admirable que la santidad mantenida desde el nacimiento.

Solemos hablar equivocadamente de la primera, de la segunda, de la tercera conversión de Pascal: no hay giros bruscos en su vida, sino más bien una continua purificación, sólo acelerada, no condicionada, por algunos acontecimientos exteriores.

En su proceso de elevación fue considerablemente ayudado por su hermana menor, Jacqueline, monja en Port-Royal, la cual, en sus acciones y en las cartas conservadas, se muestra en la comprensión del sufrimiento igual que su ilustre hermano. 
fratello.

Fino al 1654 dura il periodo mondano di Pascal; ha degli amici nell'alta società, pare fosse un giocatore accanito, e non rimase insensibile alle grazie femminili, dato che nel 1650 compose un Discours sur les passions de l'amour che è di rara profondità psicologica.

Nel 1654 sopravviene uno di quegli incidenti esteriori che accelerano la già esistente ascesi. Passando sul ponte di Neuilly i cavalli della sua vettura s'imbizzarriscono: egli rimane con la carrozza sospeso fra il ponte ed il fiume, trattenuto da una cinghia; fu salvato mezzo minuto prima che la cinghia si spezzasse. Là dove uno spirito vanitoso avrebbe visto un miracolo, cioè un segno di predilezione divina, Pascal non vide che un «avvertimento». Si ritirò dal mondo, si riavvicinò a Port-Royal, scrisse in difesa di questo le sue immortali Provinciales. Poi, martoriato da innumerevoli dolorosissime malattie, si diede a scrivere la sua opera sulla religione; gli appunti a le note preparatorie di quest'opera, la cui stesura organica non venne neppur cominciata, costituiscono le sue Pensées, che sono uno dei massimi risultati del pensiero e dell'anima umana.

Il 19 agosto 1662 morì, a trentanove anni.

\section{Il pensiero di Pascal}

Il pensiero di Pascal è tanto complesso che è indispensabile cercare di distinguere le varie componenti di una tale personalità, dopo aver sfrondato le leggende che si sono accumulate attorno a questa figura apparsa subito tanto al di sopra
Hasta el 1654 dura el período mundano de Pascal. Tiene amigos pertenecientes a la alta sociedad, y parece haber sido un jugador empedernido, no permaneciendo insensible a las gracias femeninas: de hecho, en 1650 compuso un Discurso sobre las pasiones del amor de una profundidad psicológica poco común.

En 1654 sobreviene uno de esos accidentes externos que aceleran su ya existente ascesis. Pasando sobre el puente de Neuilly los caballos de su carroza se desbocaron. Pascal permaneció en la carroza suspendido entre el puente y el río, retenido por una correa. Fue salvado medio minuto antes de que la correa se rompiera. Donde un espíritu vanidoso vería un milagro, es decir, un signo de predilección divina, Pascal no vio más que una «advertencia». Se retiró del mundo, se aproximó a Port-Royal, escribió en defensa de éste sus inmortales Provinciales. Después, martirizado por innumerables enfermedades dolorosas, se entregó a escribir su obra sobre la religión. Los apuntes y las notas preparatorias de esta obra, cuya elaboración orgánica ni siquiera fue comenzada, constituyen sus Pensamientos, que son uno de los máximos logros del pensamiento y del alma humana.

El 19 de agosto de 1662 muere Pascal, a los treinta y nueve años de edad.

\section{El pensamiento de Pascal}

El pensamiento de Pascal es tan complejo que es indispensable tratar de distinguir la variedad de componentes de tal personalidad después de haber pulido la leyenda acumulada en torno a esta figura que pareció tan por encima de los otros hombres. 
degli altri uomini.

Pascal è un ammalato. Non soltanto soffriva di tubercolosi ossea ma aveva anche una forte nevrosi: sofferenze fisiche e sofferenze morali che saltano fuori evidenti in ogni sua pagina, non fosse altro che nell'ardore febbrile, in quella fretta di apostolato che è comune a tutti coloro che avendo qualcosa da dire temono inconsciamente di non avere il tempo per farlo.

\section{Pascal è uno scienziato.} Intendo dire che ha la posizione spirituale dello scienziato, oltre che le conoscenze: anche quando lo crediamo rapito nel misticismo, lo spirito matematico, lo spirito di ricerca, l'ansia di andare al fondo delle cose lo riprendono ad un tratto. Spirito scientifico lievitato da una profonda sensibilità poetica («le silence éternel de ces espaces infinis m'effraie») che lo spinge sempre a cercare la via più ardua ma più breve per i suoi ragionamenti, ed a portare $i$ risultati raggiunti sino alle loro estreme conseguenze là dove la logica sembra (e non è) essere una sfida al nostro pedestre buon senso («il y a plus de bon sens sous le semelles des Parisiens qu'il n'y a de pensées dans leurs têtes», orgogliose parole del Santo-Matematico).

Pascal ha il temperamento dello scettico. («Je croirai qu'une mouche est une mouche lorsque l'ayant comparée avec cent autres insectes et après avoir contrôlé ma vue, je m'apercevrai qu'elle a des caractères individuels et constants.») Egli è il discepolo di Montaigne che conosce a fondo, del quale cita lunghi brani. Crede poco agli strumenti di
Pascal es un hombre enfermo. No sólo sufría una tuberculosis ósea sino que, además, padecía una fuerte neurosis: sufrimiento físico y moral que se pone en evidencia en cada una de sus páginas, aunque no era otro que el ardor febril en la urgencia de apostolado que es común a todos aquellos que teniendo algo que decir temen inconscientemente no tener tiempo para hacerlo.

Pascal es un científico. Quiero decir que tiene la posición espiritual del científico, así como los conocimientos; incluso cuando lo consideramos secuestrado en el misticismo, el espíritu matemático, el espíritu de investigación, la ansiedad por llegar al fondo de las cosas lo liberarán de repente. Espíritu científico añadido a una profunda sensibilidad poética («el silencio eterno de estos espacios infinitos me aterra» ${ }^{8}$ ), que lo empuja siempre a buscar el camino más arduo pero a su vez más breve para su razonamiento, y llevar los resultados alcanzados hasta sus últimas consecuencias allá donde la lógica parece ser (y no es) un desafío para nuestro pedestre sentido común («bajo la suela de los zapatos de los parisinos hay más sentido común que pensamientos en sus cabezas», orgullosas palabras del SantoMatemático).

Pascal tiene el temperamento del escéptico. («Creo que una mosca es una mosca que, cuando se compara con un centenar de otros insectos y después de haber controlado mi vista, observo que tiene caracteres individuales $y$ constantes»). Pascal es discípulo de Montaigne, al cual conoce a fondo y cita en largos pasajes. Cree poco en los instrumentos de indagación del hombre

8 Blaise Pascal, Pensées, L201/B206. Para la localización de los fragmentos de esta obra se utilizan en paralelo las referencias de las ediciones de Louis Lafuma (L), de 1951, y de León Brunschvicg (B), de 1897, con sus respectivas numeraciones. La traducción al español de estos fragmentos es cortesía de Gabriel Albiac. 
indagine dell'uomo («après avoir contrôle ma vue»!). I sensi «abusent la raison par des fausses apparences; et cette même piperie qu'ils apportent à l'âme, ils la reçoivent d'elle à leur tour: elle s'en revanche». Il giudizio? «Il n'y a qu'un point indivisible qui soit le véritable lieu, la perspective l'assigne dans l'art de la peinture. Mais dans la vérité et la morale qui l'assignera?» L'immaginazione? «C'est cette partie décevante dans l'homme, cette maîtresse d'erreur et de fausseté, et d'autant plus fourbe qu'elle ne l'est pas toujours.»

A fianco di queste cause immanenti di errore, vi sono delle cause secondarie, sempre presenti benché accidentali: l'abitudine, l'età, le malattie, l'interesse («merveilleux instrument pour nous crever les yeux agréablement»; «voilà pourquoi les institutions purement humaines n'ont qu'un fondement ruineux»; «Plaisante justice qu'une rivière borne. Vérité en deçà des Pyrénées, erreur au-delà»). dir di più.

Montaigne non avrebbe potuto

Pascal è un ironista. Nelle Provinciales vi sono scene di altissima commedia. Pascal è maestro nel ricercare, per porlo poi a nudo, il punto debole, il «punctum dolens», onde farlo apparire nella sua miseria a nella sua squallida comicità. Di questa facoltà d'ironia feroce egli si serve dapprima contro $\mathrm{i}$ gesuiti, ma in seguito la rivolgerà contro l'umanità intera. Motteggiatore spietato, metterà alla berlina la vanità a le piccinerie
( $i$ «espués de haber controlado mi vista»!). Los sentidos «engañan a la razón mediante falsas apariencias; y esta misma burla que aportan al alma, la reciben a su vez de ella: la razón se toma la revancha» ${ }^{9}$. ¿Y el juicio? «Sólo hay un punto indivisible que es el verdadero lugar, la perspectiva lo asigna en el arte de la pintura. Mas en la verdad y la moral ¿quién lo asignará? $\rangle^{10}$ ¿La imaginación? «Es esta parte dominante en el hombre, esta maestra de error y de falsedad, y tanto más engañosa cuanto que no siempre lo es» ${ }^{11}$.

Junto a estas causas inmanentes de error existen causas secundarias, siempre presentes aunque accidentales: el hábito, la edad, la enfermedad, el interés («un maravilloso instrumento para arrancarnos los ojos agradablemente» ${ }^{12}$; «es por ello que las instituciones puramente humanas no tienen más que un fundamento ruinoso» ${ }^{13}$; «Curiosa justicia que un río limita. Verdad de este lado de los Pirineos, error del otro lado» ${ }^{14}$ ).

Montaigne no podría haberlo dicho mejor.

Pascal es un ironista. En las Provinciales existen escenas de alta comedia. Pascal es un maestro en la búsqueda de los puntos débiles, para posteriormente dejarlos al desnudo - el «punto doliente»- con el fin de mostrar en su miseria la sórdida comicidad. Esta facultad de feroz ironía la utiliza Pascal en principio contra los jesuitas, pero inmediatamente la dirigirá contra la humanidad entera. Burla implacable que evidencia la

\footnotetext{
Ibidem, Cfr. L45/B83.

Ibidem, Cfr. L21/B381

Ibidem, Cfr. L44/B82.

Ibidem, Cfr. L44/B82.

3 Esta frase no se corresponde con una cita de Pascal sino del manual de René [Doumic, Histoire de la littérature française]. Lampedusa la introduce como comentario de las sucesivas célebres citas pascalianas (Nota del editor de la Opere de Lampedusa).

14 Blaise Pascal, Pensées, Cfr. L60/B294.
} 
dell'uomo.

Pascal è un asceta. Si rallegrava delle sue sofferenze fisiche («qui sont une augmentation de l'esprit»; verissimo... quando si è Pascal, però) e volontariamente le raddoppiava. Quella che egli ama è la fede raggiunta attraverso la sofferenza («Je méprise les douillets. Je n'approuve que ceux qui cherchent en gémissant»).

Pascal infine, è un credente. Fede duramente conquistata («je me suis jeté en désespéré aux pieds de la Croix»), ma assoluta infine $\mathrm{e}$ matematicamente sicura. Egli è forse il solo scettico che abbia trovato risposta a tutti i suoi dubbi. Non ci ha detto come perché queste cose sono ineffabili anche per un genio dell'espressione quale era lui.

E fraintenderlo completamente, è anzi insultarlo il mettere in dubbio la sincerità della sua fede. Ai pensatori del suo calibro la verità sulle proprie opinioni s'impone.

Pascal è la grande vittoria della fede sul Dubbio. La solo forse. Ma ben grande. Pascal sintetizza in sé molte antinomie e senza precedenti.

\section{Les Provinciales}

Sono diciotto lettere «de Louis de Montalte à un Provincial de ses amis et aux R.R.P.P. Jésuites sur la morale et la Politique de ces Pères»». La prima fu pubblicata il 3 gennaio 1656. L'ultima quattordici mesi dopo.

Le prime quattro sono dedicate alle liti giansenistiche, alla quistione della Grazia. A cominciare dalla quinta Pascal abbandona la tattica difensiva ed attacca con un acume ed vanidad y mezquindad del hombre.

Pascal es un asceta. Se alegraba de sus sufrimientos físicos («que suponen un acrecentamiento del espíritu» ${ }^{15}$; muy cierto... pero cuando se es Pascal) y voluntariamente los duplicaba. La que él ama es la fe alcanzada a través del sufrimiento («Desprecio a los delicados. Y no puedo aprobar más que a quienes buscan gimiendo ${ }^{16}$ ).

Pascal, por último, es un creyente. Fe duramente conquistada («me arrojé desesperadamente a los pies de la Cruz»), pero finalmente absoluta y matemáticamente segura. Él es quizás el único escéptico que encontró respuesta a todas sus dudas. Sin embargo, no nos dijo cómo, pues estas cosas son inefables incluso para un genio de la expresión cual él era.

Es completamente malintencionado, y de hecho supone un insulto, el poner en duda la sinceridad de su fe. En los pensadores de su calibre se impone la verdad a sus propias opiniones.

Pascal representa la gran victoria de la fe sobre la duda. La única tal vez. Pero bien grande. Pascal, sin precedentes, sintetiza en sí muchas antinomias.

\section{Las Provinciales}

Son dieciocho cartas «de Louis de Montalte a un provinciano por uno de sus amigos, y a los reverendos padres jesuitas sobre la moral y la política de estos padres». La primera fue publicada el 3 de enero de 1656. La ultima catorce meses después.

Las cuatro primeras cartas estás dedicadas a las disputas jansenistas, a la cuestión de la Gracia. Al comienzo de la quinta Pascal abandona la táctica

\footnotetext{
15 Blaise PASCAL, Cfr. Oración para pedir a Dios el buen uso de las enfermedades, II.

16 Blaise Pascal, Pensées, Cfr. L405/B421.
} 
un brio senza precedenti la morale dei gesuiti e la casistica. Egli immagina un padre gesuita, uomo eccellente e mite, che esponga in un dialogo le teorie più insostenibili con candore $\mathrm{e}$ bonomia. Pascal fa da interlocutore e trascina sempre più avanti l'ingenuo gesuita, finché l'assurdità appare lampante. Non vi è una contradizione della ricchissima letteratura gesuitica che non sia posta in mostra e che non sia oggetto del più urbano scherno. Un vero gesuita, le père Bouhours, lo confessa: «Nous sentîmes la terra trembler sous nous». Dalla nona lettera in poi il gesuita-capro espiatorio scompare e Pascal si rivolge direttamente all'intera Compagnia di Gesù. Le ultime due lettere trattano di nuovo della quistione della Grazia.

Les Provinciales fecero uscire le dispute teologali dal chiuso delle scuole e ne mostrarono al pubblico l'estrema importanza ed il riverbero sulla morale spicciola. E per far questo Pascal ha creato una lingua nuova, nos più rigida come quella dei dottori in teologia, non più puramente astratta come quella di Descartes, ma pieghevole, duttile, capace di rendere tutte le risonanze, che si eleva talvolta alla più alta indignazione, che scende spesso al tono della beffa amara e spietata. E che procede per mezzo di frasi corte, rapide, nervose. La lingua della polemica è stata creata da questo grande mistico.

\section{Les Pensées}

Les Provinciales non dovevano essere che un episodio della attività letteraria di Pascal. L'opera conclusiva della sua vita doveva essere 1'Apologie de la Religion chrétienne. Cominciò a lavorare defensiva y ataca la moral de los jesuitas y la casuística con una perspicacia y un brío sin precedentes. Él imagina un padre jesuita, hombre excelente y apacible, que expone en un diálogo las teorías más insostenibles con candor y bonhomía. Pascal actúa de interlocutor y refuta las exposiciones del ingenuo jesuita hasta que el absurdo parece obvio. No existe una contradicción de la riquísima literatura jesuítica que no se exhiba y que no sea objeto del mayor ridículo público. Un verdadero jesuita, el padre Bouhours, lo confiesa: "Sentimos la tierra temblar bajo nuestros pies». De la novena carta en adelante el jesuita-chivo expiatorio desaparece y Pascal se dirige directamente a toda la Compañía de Jesús. Las dos últimas cartas tratan de nuevo sobre la cuestión de la Gracia.

Las Provinciales lograron sacar de las paredes de las escuelas las disputas teologales, mostrando al público su extrema importancia y el eco sobre la moral mundana. Para hacer esto Pascal creó una nueva lengua, menos rígida que la de los doctores en teología, sin llegar a lo puramente abstracto de Descartes, sino más plegable, dúctil, y capaz de ofrecer todas las resonancias; que se eleva a veces a la más alta indignación y desciende a menudo al tono de burla amarga y despiadada, procediendo por medio de frases cortas, rápidas $\mathrm{y}$ nerviosas. La lengua de la polémica ha sido creada por este gran místico.

\section{Los Pensamientos}

Las Provinciales no dejan de ser más que un episodio de la actividad literaria de Pascal. La obra concluyente de su vida debía ser la Apología de la religión cristiana. Comenzó a trabajar en ella en torno al 1658, sin embargo, la 
intorno ad essa sin dal 1658 ma la malattia feroce gl'impediva ogni altra fatica all'infuori di quella di fissare $i$ propri pensieri, seguendo il filo delle sue meditazioni, su qualsiasi pezzetto di carta che gli capitasse fra le mani. L'autografo delle Pensées è composto da centinaia di straccetti di carta e di stoffa, coperti dalla sua scrittura febbrile ed incollati poi malamente sui fogli di un registro che è esposto adesso con grande e meritatissima pompa alla Biblioteca Nazionale di Parigi. Quel che doveva fare l'originalità dell'opera era il piano costruttivo. "Aux incroyants il faut s'adresser avec le langage de l'incroyance.» $\mathrm{E}$ da questo proposito d'indirizzarsi ai miscredenti è nata la straordinaria arditezza del famoso «pari» che a torto è stata guardata come prova di dubbio dell'autore ma che è un metodo di estrema audacia adoperato non già per far credere ma per indurre gli indolenti in materia spirituale ad occuparsi dei problemi religiosi.

Per un logico della forza di Pascal le quistioni di metodo erano importantissime e sarebbe quindi di capitale interesse conoscere la disposizione che egli aveva intenzione di dare ai suoi pensieri.

Disgraziatamente manchiamo di informazioni su questo punto; o meglio, ne abbiamo troppe e discordanti. La sorella di Pascal, Mme Périer, ci assicura di aver udito dal fratello l'esposizione dello schema logico della sua l'Apologie de la Religion. Il giansenista M. de Saci afferma aver ricevuto personalmente delle confidenze sullo stesso argomento. E le due disposizione proposte sono in netto contrasto. feroz enfermedad le impedía cualquier otro esfuerzo que no fuera el de fijar sus propios pensamientos, siguiendo el hilo de sus meditaciones en cualquier pedazo de papel que cayera en sus manos. El manuscrito autógrafo de los Pensamientos está compuesto sobre cientos de trozos de papel cosidos en legajos, cubiertos por su febril escritura, que luego fueron malamente pegados sobre grandes hojas de papel y encuadernados, y ahora se exponen con gran y merecida pompa en la Biblioteca Nacional de París. Es lo que se supone debería formar el plan constructivo original de la obra. «A los incrédulos hay que dirigirse con el lenguaje de la incredulidad». Y del propósito de orientar a los no creyentes surgió la extraordinaria osadía de la famosa «apuesta» ${ }^{17}$, que erróneamente ha sido considerada como prueba de duda del autor, siendo en realidad un método de extrema audacia utilizado no con el fin de hacer creer sino más bien para incitar a los indolentes en materia espiritual a ocuparse de los problemas religiosos.

Para un lógico de la fuerza de Pascal la cuestión del método era muy importante, y por lo tanto sería de capital interés conocer la disposición que iba a dar a sus pensamientos.

Desgraciadamente carecemos de la información adecuada con respecto a este punto; o, mejor dicho, tenemos demasiada $\mathrm{y}$, además, discordante. La hermana de Pascal, madame Périer, nos asegura que oyó del hermano la exposición del esquema lógico de la Apología de la Religión. El jansenista monsieur de Saci afirma haber recibido personalmente confidencias acerca del mismo tema. Y las dos disposiciones propuestas se encuentran en neto 
Poiché questi due testimoni sono della più grande rispettabilità ed evidentemente in buona fede si deve credere a due progetti successivi di Pascal; e quindi non possiamo esser sicuri di quale sarebbe stato preferito, o se addirittura non sarebbe stato adottato un terzo od un quarto a noi ignoti.

Del resto il danno è minimo; men che minimo anzi, perché dalla forma spezzettata e convulsa nella quale il trattato ci è giunto emana una tale sincerità, una tale violenza di convinzione che avrebbe soltanto potuto essere diminuita dall'armonia e dalla levigatezza di una composizione studiata.

Venuto meno il fine apologetico, rimane una massa colossale di pensieri di una profondità e di un'amarezza inaudita sulla «condition humaine» (questa espressione, anch'essa sovraccarica di pessimismo e passata adesso allo stato di luogo comune, è di Pascal).

Non starò a dirvi che ogni persona che voglia essere qualcosa di più un bipede implume deve leggere Les Pensées. E perciò sarebbe inutile contraste. Debido a que estos dos testigos son de la mayor respetabilidad $\mathrm{y}$, evidentemente, de buena fe, se debe creer en dos proyectos sucesivos de Pascal, y por lo tanto no podemos estar seguros de cuál podría haber sido el preferido, o de si hubiera adoptado un tercer $o$ un cuarto criterio que ignoramos.

Por lo demás, el perjuicio es mínimo; de hecho, menos que mínimo, porque de la forma dividida y convulsa en la cual el tratado ha llegado hasta nosotros emana una sinceridad, una fuerza de convicción que sólo la armonía y el pulimento de una composición estudiada podría hacer disminuir.

No habiéndose podido cumplir el fin apologético de la obra, en su lugar ésta nos ofrece un colosal conjunto de pensamientos de una profundidad y de una amargura sin precedentes sobre la «condición humana» (esta expresión, también sobrecargada de pesimismo y que ahora se ha constituido en tópico, es de Pascal) ${ }^{18}$.

No voy a decir que toda persona que quiera ser algo más que un bípedo implume debe leer Los Pensamientos.

18 Hay dos fragmentos en las ediciones de los Pensées en donde aparecen expresiones equivalentes en significado a la «condition humaine»: L24/B127 («Condition de l'homme. Inconstance, ennui, inquiétude», es decir, «Condición del hombre. Inconstancia, hastío, inquietud»); L434/B199 («Qu'on s'imagine un nombre d'hommes dans les chaînes, et tous condamnés à la mort, dont les uns étant chaque jour égorgés à la vue des autres, ceux qui restent voient leur propre condition dans celle de leurs semblables, et, se regardant les uns et les autres avec douleur et sans espérance, attendent à leur tour. C'est l'image de la condition des hommes», esto es, «Imaginemos a un número de hombres encadenados y todos ellos condenados a muerte, algunos de los cuales siendo cada día degollados a la vista de los otros, los que quedan ven su propia condición en la de sus semejantes, y, mirándose unos a otros con dolor y sin esperanza, esperan su turno. Es la imagen de la condición humana»).

Del fragmento L434/B199 no se conserva el original sino las dos copias posteriores. La última frase de este fragmento ( $C$ 'est l'image de la condition des hommes) no es de Pascal. Se trata de un añadido a la Primera copia (la Segunda copia no contiene este añadido), como un resumen del fragmento, de la mano de Etienne Périer para la edición de 1678, según indican Descotes y Proust en su edición digital de los Pensées (www.penseesdepascal.fr/Loi/Loi.php). Sin embargo, esta expresión de "la imagen de la condición humana" ha tenido una gran resonancia en la historia de la literatura moderna, inspirando a escritores como el propio Lampedusa o André Malraux, el cual adoptó la adición realizada por Périer para dar título a su famosa novela de 1933, La condición humana.

Como se desprende de varios fragmentos relacionados de los Pensées (v. gr., L131/B434), esta alegoría de la "condición humana" está asociada con aquellos temas en los que Pascal muestra la situación trágica a la que se verá abocada la miseria del hombre sin Dios. 
trascriverne qualcuna. Però non vorrei lasciarvi andar via con la mia prosa nelle orecchie quando potete invece ascoltare qualcuna di queste «poignantes»e talvolta sconvolgenti enunciazioni:

«Le soleil ni la mort ne se peuvent regarder fixement».

«Le dernier acte est sanglant, quelque belle que soit la comédie en tout le reste; on tire un drap sur le visage, et tout est dit».

«Les hommes n'ayant pu guérir la mort, la misère, l'ignorance, ils se sont avisés, pour se rendre heureux, de n'y point penser».

«La condition humaine ici-bas est misérable, et la tristesse y est si parfaitement inhérente, que l'homme ne saurait en supporter la connaissance et rester en repos en face de soi. Le jeu, la chasse, le travail du savant, les occupations du magistrat, du conquérant, du roi, tous ces biens prétendus n'ont d'autre utilité que de détourner l'homme de lui-même et de le "divertir"».

Questo è il pessimismo pascaliano. Ma vi è anche il suo aspetto ottimista, non certo ridanciano, ma quell'ottimismo che può provenire da un alto senso della dignità umana: «L'homme connaît qu'il est misérable; Il est donc misérable puisqu'il l'est; mais il est
Así que sería inútil transcribir algunos. Sin embargo, no quisiera dejaros ir con mi prosa en los oídos cuando en su lugar se pueden escuchar algunos de estos «punzantes» y a veces estremecedores enunciados:

«Ni al sol ni a la muerte se les puede mirar fijamente» ${ }^{19}$.

«El último acto es sangriento, por muy bella que sea la comedia en todo lo demás; se extiende un paño sobre el rostro, $\mathrm{y}$ todo está dicho» ${ }^{20}$.

«Los hombres no habiendo podido curar la muerte, la miseria, la ignorancia, se han concertado, para hacerse felices, en no pensar en ello» ${ }^{21}$.

«La condición humana en este mundo es miserable, y la tristeza es tan perfectamente inherente que el hombre no puede soportar el conocimiento y permanecer en reposo frente a sí. El juego, la caza, el trabajo del sabio, las ocupaciones del magistrado, el conquistador, el rey, todos estos bienes pretendidos no tienen otra utilidad que distraer al hombre de sí mismo y la "diversión"»" ${ }^{22}$.

Éste es el pesimismo pascaliano. Mas también existe su aspecto optimista - no muy risueño, ciertamente-, el cual provendría de un alto sentido de la dignidad humana: «El hombre conoce que es miserable. Es pues miserable, puesto que lo es, pero es muy grande puesto que lo conoce» ${ }^{23}$.

19 La cita no pertenece a Los Pensamientos sino a las Máximas de La Rochefoucauld. (Nota del editor de la Opere de Lampedusa).

20 El editor de la Opere de Lampedusa indica que, aunque la cita no es la correcta, la recoge tal cual ya que formaba parte del conjunto de máximas que constituían la guía (stella polare) de Giuseppe Tomasi. Y añade la cita correcta, que es la siguiente: «Le dernier acte est sanglant, quelque belle que soit la comédie en tout le reste. On jette enfin de la terre sur la tête et en voilà pour jamais» (El último acto es sangriento, por muy bella que sea la comedia en todo lo demás. Se arroja finalmente tierra sobre la cabeza y se acabó para siempre) Blaise Pascal, Pensées, L165/B210.

21 Blaise Pascal, Pensées, L133/B168.

22 Este texto no es una cita de Pascal sino un resumen del apartado «Le divertissement», legajo VIII de los Pensamientos. (Nota del editor de la Opere de Lampedusa).

Para mayor detalle ver L136/B139, que contiene lo esencial de la teoría pascaliana acerca de la "diversión" como autoengaño del hombre.

23 Blaise Pascal, Pensées, Cfr. L122/B416. 
bien grand puisqu'il le connaît». «L'homme n'est qu'un roseau, le plus faible de la nature; mais c'est un roseau pensant. Il ne faut pas que l'univers entier s'arme pour l'écraser: une vapeur, une goutte d'eau suffit pour le tuer. Mais quand l'univers l'écraserait, l'homme serait encore plus noble que ce qui le tue, puisqu'il sait qu'il meurt et l'avantage que l'univers a sur lui, l'univers n'en sait rien».

Il contrasto fra la miseria dell'uomo ed il suo valore è l'enigma al quale, secondo Pascal, la sola risposta è Dio. «Quelle chimère est $\square$ ce donc que l'homme? Quelle nouveauté, quel monstre, quel chaos, quel sujet de contradiction, quel prodige! Juge de toutes choses, imbécile ver de terre, dépositaire du vrai, cloaque d'incertitude et d'erreur, gloire et rebut de l'univers»... «La nature confond les pyrrhoniens, et la raison confond les dogmatiques». «Toutes ces misères-là même prouvent sa grandeur. Ce sont misères de grand seigneur, misères d'un roi dépossédé»».

Mai si erano udite prima, mai più udiremo simili grida di angoscia.

Per uscire dal tormento, Pascal ha trovato Dio. «C'est le cœur qui sent Dieu, et non la raison. Voilà ce que c'est que la foi: Dieu sensible au coeur». E, dopo, subito, il pensiero supremo: «Tu ne me chercherais pas, si tu ne m'avais trouvé».
«El hombre no es más que una caña, la más débil de la naturaleza, pero es una caña pensante. No hace falta que el universo entero se alce en armas para aplastarlo; un vapor, una gota de agua bastan para matarlo. Mas, aun cuando el universo lo aplastara, el hombre seguiría siendo más noble que lo que lo mata, puesto que él sabe que muere y sabe la ventaja que el universo tiene sobre él. El universo nada sabe de ello» ${ }^{24}$.

El contraste entre la miseria y la grandeza del hombre es el enigma ante el cual, según Pascal, la única respuesta es Dios. "¿Qué quimera es, pues, el hombre? ¿Qué novedad, qué monstruo, qué caos, qué sujeto de contradicciones, qué prodigio? Juez de todas las cosas, imbécil gusano de tierra, depositario de lo verdadero, cloaca de incertidumbre y de error, gloria y escoria del universo»... «La naturaleza confunde a los pirronianos y la razón confunde a los dogmáticos» ${ }^{25}$. «Todas esas miserias mismas prueban su grandeza. Son miserias de gran señor. Miserias de un rey desposeído» ${ }^{26}$.

Nunca se habían oído antes, nunca más oiremos semejantes gritos de angustia.

Para salir del tormento, Pascal ha encontrado a Dios. «Es el corazón quien siente a Dios y no la razón. He ahí lo que es la fe. Dios sensible al corazón, no a la razón» ${ }^{27}$. Y, después, de inmediato, el pensamiento supremo: «Consuélate. Tú no me buscarías si no me hubieses encontrado» ${ }^{28}$.

Ibidem, Cfr. L200/B347.
Ibidem, Cfr. L131/B434.

Ibidem, Cfr. L131/B
Ibidem, L116/B398.

Ibidem, L424/B278. En la transcripción de Lampedusa se observan cambios de puntuación y la omisión de la última frase del fragmento (non à la raison), que incluimos en la traducción. La cita completa en la edición de Lafuma es la siguiente: «C'est le cœur qui sent Dieu et non la raison. Voilà ce que c'est que la foi. Dieu sensible au cœur, non à la raison».

28 Se corresponde con una frase del fragmento L919/B553 y 791 de los Pensées de Pascal, dentro de los fragmentos no registrados por la copia. Fue publicado por Faugère en 1844. En la transcripción de Lampedusa se observan cambios de puntuación y la omisión de la primera palabra (Console-toi), que incluimos en la 
Tutto, passando attraverso la mente di Pascal, prende un aspetto diverso. Montaigne parla come lui della miseria e della ignoranza umana; ma se ne diverte. Pascal ne soffre. La sua mentalità è drammatica: le Pensées sono un dramma, uno dei più alti, la lotta della fede contro il dubbio, e la forma dialogata viene spesso spontanea alla sua mente. «Je pensais à toi dans mon agonie, j'ai versé telle gouttes de sang pour toi».

Dello stile è inutile parlare: idea e stile qui sono la medesima cosa, come la luce ed il sole.

Parecchi pensieri sono del resto dedicati al modo di scrivere. Tutti impongono la necessità della naturalezza: «La vraie éloquence se moque de l'éloquence». "Quand on voit le style naturel on est tout étonné et ravi, car on s'attendait de voir un auteur et on trouve un homme».

Mi sono riletto circa la metà delle Pensées; e davvero preferisco tacere. «Seul le silence est grand; tout le reste est faiblesse.»

Lasciamolo dunque solo, Pascal, sulla sua cima scabra più alta di qualsiasi montagna. Lasciamolo solo, dilaniato dal duplice uragano della Fede e del Dubbio.
Todo toma un aspecto diferente cuando pasa por la mente de Pascal. Montaigne habla como Pascal de la miseria y de la ignorancia humana, pero él se divierte; Pascal sufre. Su mentalidad es dramática. Los Pensamientos son un drama; uno de los más altos: la lucha de la fe contra la duda, y la forma de diálogo espontáneo surge a menudo en su mente. «Pensé en ti en mi agonía; he vertido gotas de sangre por ti $\rangle^{29}$.

Del estilo es inútil hablar: idea y estilo aquí son la misma cosa, como la luz y el sol.

No obstante, varios de los pensamientos están dedicados al modo de escribir. Todos imponen la necesidad de la naturaleza: «La verdadera elocuencia se burla de la elocuencia» ${ }^{30}$. «Cuando se ve el estilo natural queda-mos asombrados y extasiados, porque esperábamos ver un autor y encontramos a un hombre» ${ }^{31}$.

He releído aproximadamente la mitad de los Pensamientos; y realmente prefiero callar. «Sólo el silencio es grande; todo lo demás es debilidad».

Dejemos, pues, a Pascal, solo, situado en su escabrosa cima, más alta que cualquier montaña. Dejémoslo solo, desgarrado por el doble huracán de la fe y de la duda.

FINE

FIN

traducción. La cita completa en la edición de Lafuma es la siguiente: «Console-toi, tu ne me chercherais pas si tu ne m'avais trouvé».

29 Se corresponde con la frase siguiente del fragmento citado en la anterior nota de los Pensées, L919/B553 y 791. En la transcripción de Lampedusa se observan cambios de puntuación. La cita correcta, según la edición de Lafuma, es la siguiente: «Je pensais à toi dans mon agonie; j'ai versé telle gouttes de sang pour toi».

30 Blaise Pascal, Pensées, Cfr. con el comienzo del fragmento L513/B4.

31 Ibidem, L675/B29. 